\title{
Dinámicas del bienestar animal en la India: la prohibición de Jallikattu
}

\author{
Maya Alvisa Barroso ${ }^{1}$ \\ USAL - C.A.R.I.
}

Como señala la FAO (2009), cada vez más, los distintos gobiernos, los organismos de financiación y el sector privado van incorporando la "responsabilidad social" por el medio ambiente, la justicia social, el bienestar de los animales, etc., en sus políticas y prácticas.

El documento agrega que, al integrar el bienestar de los animales en sus programas, la FAO no debería tratar el tema del bienestar animal como una cuestión independiente, sino como uno de los muchos objetivos que persigue. En particular, sostiene que las prácticas de bienestar animal necesitan ser integradas y contribuir a programas más amplios, que permitan mejorar la producción ganadera, la sanidad animal, la seguridad de los productos, la seguridad de los trabajadores y el desarrollo humano, en un marco de respeto al medio ambiente y las tradiciones culturales.

En línea con esos planteos, pueden mencionarse los denominados Objetivos de Desarrollo Sostenible (SDG), que constituyen un conjunto de 17 metas globales con 169 objetivos entre ellos. Estos objetivos figuran en la Resolución A / RES / 70/1 de las Naciones Unidas, del 25 de septiembre de 2015 y surgen de un proceso deliberativo de sus 194 Estados Miembros, así como de la sociedad civil del mundo. Los 17 Objetivos son: 1) Erradicar la pobreza, 2) Hambre cero, alcanzando la seguridad alimentaria, 3) Asegurar una buena salud y bienestar para todos, 4) Educación de calidad, 5) Equidad de género, 6) Asegurar la disponibilidad y administración sustentable de agua y saneamiento, 7) Asegurar el acceso a energías limpias que puedan costearse, 8) Trabajo decente y crecimiento económico, 9) Industria, innovación e infraestructura, 10) Reducir las desigualdades, 11) Hacer ciudades y comunidades sostenibles, 12) Asegurar un consumo y producción responsables, 13) Desarrollar acciones urgentes para afrontar el cambio climático, 14) Conservar y hacer un uso sostenible de los océanos, mares y recursos marinos, 15) Proteger, restaurar y promover el uso sostenible de ecosistemas terrestres, desarrollar un manejo sustentable de ecosistemas terrestres y bosques, combatir la desertificación y detener y revertir la degradación de las tierras y la pérdida de biodiversidad, 16) Promover la paz, la justicia e instituciones fuertes y 17) Fortalecer los medios de aplicación y revitalizar la asociación mundial para el desarrollo sostenible (Programa de las Naciones Unidas para el Desarrollo en Argentina, s.f.).

Por otro lado, es sabido que la sociedad civil ha ido desarrollando a lo largo de la historia distintas iniciativas que buscan el bienestar animal. Ese movimiento encuentra raíces históricas lejanas como las acciones del Emperador

\footnotetext{
${ }^{1}$ Maya Alvisa Barroso es Licenciada en Estudios Orientales y Doctoranda en Historia (Universidad del Salvador). Docente e investigadora, autora de distintas publicaciones, es también miembro del Grupo de Trabajo sobre India, del Comité de Asuntos Asiáticos, del Consejo Argentino para las Relaciones Internacionales (C.A.R.I.).
} 
Ashoka (S. III AC, India), que tras su conversión al Budismo, desarrolló políticas basadas en los 10 principios budistas, que derivaron en la publicación de 14 edictos, asociados a esa lógica y que propendían al bienestar animal, reemplazando la "conquista por la fuerza" por la "conquista por la justicia/lo correcto" (Basham, 1982; New World Encyclopedia, 2016); también la argumentación del filósofo Jeremy Bentham por un mejor tratamiento de los animales sobre la base de su capacidad de sentir placer y dolor (Bentham, 1780/2010), etc.

De igual modo, los diversos países han desarrollado (y continúan haciéndolo) distintas leyes que atienden la cuestión del bienestar animal y las penas consideradas para quien los daña. En el caso de la India, pueden mencionarse entre los elementos de su marco legal:

The Prevention of Cruelty Animals Act,1960,

The Performing Animals Rules, 1973 and The Performing Animals (Registration) Rules, 2001,

The Prevention Of Cruelty To Draught And Pack Animals Rules, 1965,

The Transport of Animals Rules, 1978,

Transport of Monkeys,

Transport of Cattle,

Transport of Equines,

Transport of Sheep and Goats,

Transport of Poultry by Rail, Road and Air,

Transport of Pigs by Rail or Road,

The Prevention of Cruelty to Animals (Slaughter House) Rules, 2001,

The Experiments On Animals (Control And Supervision) Rules, 1968,

The Wild Life Protection Act, 1972, etc.

Con ese panorama dado, numerosos tamiles en todo el mundo (el estado de Tamil Nadu posee más de 60 millones de personas y un buen número de emigrados) se unieron para protestar contra la prohibición de la Corte Suprema (2014) contra Jallikattu, actividad que se autodenomina deporte -al que se le atribuyen 2000 años de antigüedad-, en el que se "doma” un toro, durante las celebraciones de Pongal, festival tamil de la cosecha.

La protesta popular en apoyo al desarrollo de la festividad, recibió también el apoyo de figuras conocidas de las artes locales (Kollywood) y alcanzó incluso los dichos del Primer Ministro Narendra Modi, acerca de que se realizaban esfuerzos para poder cumplir las aspiraciones culturales de las personas. Considerando entonces esa acción positiva del gobierno central, el Gobernador de Tamil Nadu, Vidyasagar Rao (BJP), aprobó la ordenanza que permite la realización de Jallikattu en 2017. 
La primera prohibición contra Jallikattu se produjo en marzo de 2006, como decisión del Alto Tribunal de Madrás. Entre 2009 y 2014, distintas instancias estatales se expidieron, continuando con la prohibición. En enero de 2016, el Ministerio de Ambiente, Bosques y Cambio Climático enmendó su notificación de 2011, abriendo el camino para la realización de Jallikattu en los estados de Maharashtra, Karnataka y Gujarat, con condiciones específicas. Sin embargo, esa acción fue desafiada por PETA, el Animal Welfare Board of India (AWBI) y otras organizaciones ante la Corte Suprema del país, que apoyan la prohibición de la celebración, sobre la base de que la crueldad no se limite al acto de matar, sino que incluya el sufrimiento innecesario y la tortura inducida en animales para el entretenimiento humano, enfatizando el rol del Estado y sus ciudadanos de asegurar el bienestar de los animales. En julio de 2016, la Corte Suprema prohibió Jallikattu nuevamente (Firstpost, 2017; Lakshmana, 2017; Safi, 2017; The Indian Express, 2017; Vyawahare, 2017).

Finalmente, confluyendo numerosos factores sobre la celebración de Jallikattu, relativos a cuestiones género, casta, autopercepción de los tamiles, elementos mentados como "tradicionales", la participación de agentes sociales "extranjeros" como PETA, etc., cabe también agregar que esos debates se dan en un contexto nacional de prohibición de la matanza de vacas en la mayoría de los estados indios y del consumo de su carne, mientras el país es el principal exportador de carne vacuna del mundo.

En tal sentido, se requiere un debate verdaderamente más profundo sobre las cuestiones relativas al bienestar animal, en el segundo país más poblado del planeta (lo cual inevitablemente incide en la dinámica humanos-animales y sus respectivos hábitats), intentando deslindar la gran cantidad de elementos culturales asociados a ello, en la búsqueda de poder asegurar, efectivamente, el bienestar de los seres vivos no humanos, afianzando el espacio de desarrollo humano que ello implica.

\section{Referencias Bibliográficas:}

BASHAM, A. L. (1982). Asoka And Buddhism - A Reexamination: Presidential Address Given on the Occasion of the Fourth Conference of the IABS Madison, Wisconsin, August, 1980. The Journal of the International Association of Buddhist Studies, 5(1), 131-143. Recuperado de:

https://journals.ub.uni-heidelberg.de/index.php/jiabs/article/viewFile/8572/2479

BENTHAM, J. (1780/2010). An Introduction to the Principles of Morals and Legislation. Recuperado de http://www.earlymoderntexts.com/assets/pdfs/bentham1780.pdf 
FAO/FOOD AND AGRICULTURE ORGANIZATION OF THE UNITED NATIONS (2009). Capacity building to implement good animal welfare practices. Report of the FAO Expert Meeting. FAO Headquarters (Rome), 30 September -3 October 2008. Roma: FAO. Recuperado de http://www.fao.org/fileadmin/user upload/animalwelfare/i0483e00 1.pdf LAKSHMANA, K. V. (2017). "Jallikattu ban: Thousands continue protests in Chennai's Marina Beach". Hindustan Times (18 de enero). Recuperado de http://www.hindustantimes.com/india-news/iallikattu-ban-thousands-continueprotests-in-chennai-s-marina-beach/story-VOWa0A6zHIUgY9pAmiVrnN.html

NEW WORLD ENCYCLOPEDIA (2016). Ashoka. Recuperado de http://www.newworldencyclopedia.org/entry/Ashoka

PROGRAMA DE NACIONES UNIDAS PARA EL DESARROLLO EN ARGENTINA (s.f.). Agenda de desarrollo post-2015. Recuperado de http://www.ar.undp.org/content/argentina/es/home/post-2015/sdg-overview.html

SAFI, M. (2017). "Tamil Nadu passes order to lift bull-taming ban after angry protests". The Guardian (23 de enero). Recuperado de https://www.theguardian.com/world/2017/jan/23/tamil-nadu-passes-order-lift-jallikattu-bull-taming$\underline{\text { ban-india-protests }}$

SUDHIR, T. S. (2017). "Jallikattu ban: Peta receives abusive calls, threat of rape; says fight for animal rights will continue". Firstpost (21 de enero). Recuperado de http://www.firstpost.com/india/jallikattu-ban-peta-receivesabusive-calls-threats-of-rape-says-fight-for-animal-rights-will-continue-3213780.html

THE INDIAN EXPRESS (2017). "Jallikattu protests: What is the uproar in Tamil Nadu all about?" (13 de febrero). Recuperado de http://indianexpress.com/article/india/iallikattu-protests-modi-ordinance-chennai-marina-beachmadurai-panneerselvam-4481218/

VYAWAHARE, M. (2017). "Jallikattu reflects poorly on India: PETA India CEO after "bullying". Hindustan Times (24 de enero). Recuperado de http://www.hindustantimes.com/india-news/cruelty-to-animals-is-anti-india-says-peta-indiaceo-amid-jallikattu-protests/story-kJgbed7mmFiMPoq9vB8oSO.html

\section{Documentos}

THE PREVENTION OF CRUELTY TO ANIMALS ACT, 1960 (59 of 1960) As amended by Central Act 26 of 1982. Recuperado de http://www.envfor.nic.in/legis/awbi/awbi01.pdf

PERFORMING ANIMALS RULES, 1973. Recuperado de http://www.envfor.nic.in/legis/awbi/awbi06.html 
THE PERFORMING ANIMALS (REGISTRATION) RULES, 2001. Recuperado de http://www.fiapo.org/wpcontent/uploads/2015/03/Performing-Animals-Registration-Rules.pdf

TRANSPORT OF ANIMALS RULES, 1978. Recuperado de http://www.envfor.nic.in/legis/awbi/awbi07.pdf INDIAN STANDARD. Transport of Monkeys by Rail, Road, Air and Sea - Code of Practice (Second Revision). Recuperado de http://www.standardsbis.in/Gemini/scoperef/SR3059.pdf

NATIONAL COMMISSION ON CATTLE, Government of India (2002). Report of the National Commission on Cattle. En Department of Animal Husbandry, Dairying \& Fisheries. Recuperado de:

https://web.archive.org/web/20131110134335/http://www.dahd.nic.in/dahd/reports/report-of-the-nationalcommission-on-cattle/chapter-i-introduction.aspx

THE PREVENTION OF CRUELTY TO ANIMALS (Slaughter House) Rules, 2001. Recuperado de: http://wgbis.ces.iisc.ernet.in/biodiversity/Environ sys/legis/anirule5.htm

THE EXPERIMENTS ON ANIMALS (Control And Supervision) Rules, 1968. Recuperado de: http://www.envfor.nic.in/legis/awbi/awbi04.html

THE WILDLIFE (PROTECTION) ACT, 1972 (No. 53 of 1972) (9th September, 1972). Recuperado de http://nbaindia.org/uploaded/Biodiversityindia/Legal/15.\%20Wildlife\%20(Protection)\%20Act,\%201972.pdf 